\title{
Decentralised Static Output Feedback Stabilisation and Synchronisation of Networks *
}

\author{
Prathyush P Menon ${ }^{\text {a }}$, Christopher Edwards ${ }^{\mathrm{a}}$,

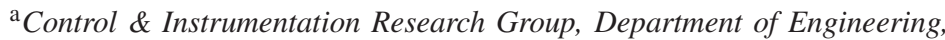 \\ University of Leicester, Leicester LE1 7RH, UK
}

\begin{abstract}
In this paper global stabilisation of a complex network is attained by applying local decentralized output feedback control to a minimum number of nodes of the network. The stabilisation of the network is treated as a rank constrained problem. Strict positive realness conditions on the node level dynamics allow nonlinearities/uncertainties which satisfy sector conditions to be considered. A network of Chua oscillators with 75 nodes is considered to demonstrate the efficacy of the approach.
\end{abstract}

Key words: decentralised control, complex networks, LMIs, output feedback

\section{Introduction}

The increasing number of control applications involving sensor arrays, cooperative unmanned air vehicles, formations of satellite systems, etc. have spotlighted problems associated with the control of network systems. A significant research problem in such applications is answering how the multiple dynamical systems operating over a network can achieve global stabilisation or performance. Many researchers have contributed to the area of control of network systems/cooperative control (see $[1,2]$ for an overview). In comparison with conventional control problems, the control of networks is much more demanding. A key issue is how the information topology of the network distribution can be exploited in the problem since the topology of the network and its associated connectivity plays an important role in determining the dynamical behaviour of the networked system. A detailed account of the development and present status of complex network theory is provided in [3-6]. A general scale-free dynamical network model was discussed in [7], and subsequently, conditions for synchronisation of such networks were derived [8]. In Ref.[9], the V-stability concept was introduced for such systems. The state feedback results in [9] depend on establishing a common Lyapunov function for studying so-called 'pinning' of complex networks. Passivity concepts have been used in [10] to study the coordination of dynamical systems in a group. In [10]

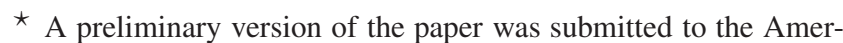
ican Control Conference 2008. Corresponding author, P. Menon, Tel. +44-116-2522874. Fax +44-116-2522619

Email addresses: ppm6@le.ac.uk (Prathyush P Menon), chris.edwards@le.ac.uk (Christopher Edwards).
}

the difference between the output variables of individual dynamical systems in a group is controlled to belong to a defined compact set, and studied as a set stability problem.

An approach based upon graph and system theoretic methods has been investigated in [12]. The paper makes use of convexity properties to describe how state agreement is achieved. Provided the systems move toward the convex hull of a set of systems, then state agreement can be achieved. In [13] this work was further investigated for state agreement/synchronisation of continuous time coupled nonlinear systems and the rendezvous problem of mobile robots. Ref. [11] focuses on stabilisation of formations of vehicles with linear dynamics, with a full order decentralised controller.

Decentralised control has a rich history in the control systems literature [33]. A decentralised adaptive output feedback controller was developed in [26] for an automated highway system, i.e. for a string of dynamical systems. The same problem was revisited in [30] using an approach based on decentralised output regulation of neutrally stable exosystems. Decentralised robust output feedback control for certain classes of nonlinear large scale interconnected systems were developed in $[28,29,14]$ and the references therein. Decentralised output feedback control of interconnected systems often appears in formation control problems and early work in this field was reported in [30]. More recently, in [31], decentralised tracking control laws have been developed for formation flying based on contraction analysis principles posed as a synchronization problem. Robust and adaptive control methods are employed in [32] to achieve synchronization for an uncertain dynamical network with an unknown but bounded nonlinear function. In this paper a particular representation of the scale-free dynamical network in 
a modified form will be investigated [7] and decentralised output feedback control will be considered. Making use of local control strategies is important from the perspective of limited computing power and sensing capability. For example, one of the challenges in the use of wireless networks comes from the limited energy of the individual systems. The objective of this paper is to stabilize the network in a locally decentralized manner using only output feedback control based on a few nodes of the network. Compared with the usual state feedback policies, only output information will be utilised for stabilizing the network, which is advantageous from the perspective of minimising sensor requirements.

The main contributions of the paper are three-fold: Firstly the whole analysis is based on a transformation using spectral decomposition to achieve an equivalent decoupled structure. This restricts the analysis of the networked dynamical system to the node level dynamics of the network. Then by employing a four block representation and an invariance structure on the networked dynamical systems, a framework is proposed for the stabilisation of the network. Secondly a decentralised output feedback strategy is proposed which uses only a subset of nodes of the network to inject the control signals. The solution is formulated in terms of a two-stage Linear Matrix Inequality (LMI) optimization procedure. The first stage of the LMI synthesis provides a sufficient condition for stabilisation: this is a convex optimization problem. The second LMI problem involves a rank constraint to obtain the minimum number of nodes for control signal injection and hence is non-convex. This is a unique feature of the method developed in this paper. In the case of a network with $N$ nodes, the selection of $k$ out of $N$ nodes for control injection is associated with a selection complexity of $\mathscr{O}\left(C_{N}^{k}\right)$. From this perspective the rank constrained stabilisation approach reported in this paper has clear significance. Thirdly the paper demonstrates the possibilities of exploiting positive realness in the closed loop node level dynamics so that the formulation can handle a certain (wide) class of nonlinearities/uncertainties satisfying sector conditions.

The expression $\mathscr{C}$ ol (.) defines a column vector and $\mathscr{D}$ iag(.) defines a diagonal matrix. $\mathbf{S}_{+}^{n}$ denotes the set of positive definite matrices. The symbols $\mathscr{N}(\cdot)$ and $\mathscr{R}(\cdot)$ represent the null space and range space of a matrix.

\section{Preliminaries}

The interconnected network dynamical system considered in this paper consists of $N$ identical dynamical elements indexed $1,2, \ldots, N$. Each vertex/node represents an $n$-dimensional dynamical system. The nodes are assumed to be coupled linearly and diffusively[8]. As and where there is an interconnection between any two dynamical systems, it constitutes an edge connecting those nodes. These interconnections are assumed to be bidirectional and hence the network is considered as a static undirected graph. The dynamics of the $i^{t h}$ node of the graph $\mathscr{G}$ are given by

$\dot{x}_{i}=A x_{i}+B u_{i}-\sum_{j=1}^{N} c L_{i j} \Gamma x_{j}+f_{i}\left(x_{i}\right)$

$y_{i}=C x_{i}$ where $x_{i} \in \mathbb{R}^{n}$ represents the $n$-dimensional state vector of the $t^{t h}$ node. The symbol $x$ represents the collective state $x=$ $\mathscr{C}$ ol $\left(x_{1}, \ldots, x_{i}, \ldots, x_{N}\right)$. The matrices $A \in \mathbb{R}^{n \times n}, B \in \mathbb{R}^{n \times m}$ and $C \in \mathbb{R}^{p \times n}$ represent the nominal linear part of the system comprising the dynamics of the $i^{t h}$ node. The signals $u_{i} \in \mathbb{R}^{m}$ and $y_{i} \in \mathbb{R}^{p}$ represent the control input and the measured outputs of the $i^{\text {th }}$ node respectively where $p \geq m$. Without loss of generality, it is assumed that the matrices $B$ and $C$ have full column and row rank respectively. The triplet $(A, B, C)$ is assumed to be a minimal realization of the $i^{t h}$ node of the network. $L \in \mathbb{R}^{N \times N}$ denotes the connectivity of the topology of the network being considered. If there is a connection between node $i$ and node $j$, then $L_{i j}=L_{j i}=-1$; otherwise $L_{i j}=L_{j i}=0$. The diagonal elements $L_{i i}=k_{i}, i=1,2, . ., N$ where $k_{i}$ is the degree of the node, defined as the number of connection incidents at the $i^{\text {th }}$ node. The Laplacian $L(\mathscr{G})$ is always rank deficient and positive semi-definite[19]. The matrix $\Gamma=\tau_{i j} \in \mathbb{R}^{n \times n}$ represents the local coupling configuration among the states of the nodes. Here it is assumed $\Gamma=\mathscr{D} \operatorname{iag}\left[\tau_{1}, \tau_{2}, . ., \tau_{i}, . ., \tau_{n}\right]$, and all the entries are 1 or 0 . The real constant $c>0$ is the coupling strength between the $i^{\text {th }}$ and $j^{\text {th }}$ node. The functions $f_{i}\left(x_{i}\right)$, represent the nonlinear parts of the dynamical system and are assumed to satisfy certain sector bounds which will be precisely defined later in the paper.

Assumption 1 The coupling strength $c$ is identical for all the connections between the nodes.

Assumption 2 The configuration matrix satisfies $\operatorname{rank}(\Gamma)=$ $m$. By rearrangement of the states of the dynamics of each node, it is possible to ensure without any loss of generality that $\Gamma$ consists of the block diagonal matrices: $\Gamma=\mathscr{D}$ iag $\left[I_{m}, 0\right]$.

Assumption 3 The configuration matrix $\Gamma$ and the input distribution matrix $B$ satisfy the geometrical constraint $\mathscr{N}(\Gamma) \cap \mathscr{R}(B)=\{0\}$.

Assumption 4 There exists an $F \in \mathbb{R}^{m \times p}$ s.t. $\Gamma=B F C$.

Remark 1 Assumption 4 implies the range space of the coupling configuration matrix is constrained within a subspace of the range space of the control input. Intuitively this ensures the control signals can be injected into the states of a particular node in the same channels as the interactions.

Assumption 5 The system $(A, B, F C)$ is controllable and minimum phase.

Lemma 1 If Assumptions 3 and 4 hold, then FCB is full rank.

Proof: From the dimensions of $\Gamma$ and $B$, it follows that $\operatorname{rank}(\Gamma B) \leq m$. Consider the homogeneous linear equation $\Gamma B \eta=0$ where $\eta \in \mathbb{R}^{m}$. Considering this equation as $\Gamma(B \eta)=0$, it is clear that the vector $B \eta \in \mathscr{N}(\Gamma)$ and $B \eta \in \mathscr{R}(B)$. By Assumption 3, $\mathscr{N}(\Gamma)$ and $\mathscr{R}(B)$ are disjoint and hence $B \eta=0$. However since by assumption $B$ is full column rank, $B \eta=0$ implies $\eta=0$. Then the only solution to $\Gamma B \eta=0$ is $\eta=0$, which means $\Gamma B$ has full column rank and therefore $\operatorname{rank}(\Gamma B)=m$. From Assumption 4 there exists a design parameter matrix $F \in \mathbb{R}^{m \times p}$ such that $\Gamma=B F C$ holds. Multiplying both sides on the right by the 
matrix $B$ and using the result that $\operatorname{rank}(\Gamma B)=m$, it follows $\operatorname{rank}(B F C B)=m$. Since

$$
\operatorname{rank}(\Gamma B)=\operatorname{rank}(B F C B) \leq \min \{\operatorname{rank}(B), \operatorname{rank}(F C B)\}
$$

it follows $\operatorname{rank}(F C B)=m$ since if $\operatorname{rank}(F C B)<m$ the inequality above implies $\operatorname{rank}(\Gamma B)<m$, which is a contradiction. Therefore $F C B$ is full rank.

The following outlines the problem to be addressed in the remainder of the paper.

Problem 1 Given a network of $N$ identical distributed $d y$ namical systems, connected according to a certain arbitrary graph $\mathscr{G}$, as described in (1) and (2), develop a systematic approach to stabilise/synchronise globally the network using a decentralised static output feedback control strategy for a certain number, $l$, of the dynamical systems, where $l$ is a positive integer strictly less than $N$.

\section{Network stabilisation: Linear case}

Consider initially the linear system described by

$\dot{x}_{i}=A x_{i}+B u_{i}-\sum_{j=1}^{N} c L_{i j} \Gamma x_{j}$

$y_{i}=C x_{i}$

subject to Assumptions 1, 2, 3, 4 and 5. From Lemma 7 it follows $\operatorname{rank}(F C B)=m$ and by assumption the triple $(A, B, F C)$ is minimum phase. It follows there exists a mapping $x \mapsto \tilde{T} x$ transforming the node state coordinates such that the triple $(A, B, F C)$ has the following special 4 block partitioned form [15]:

$A=\left[\begin{array}{ll}A_{11} & A_{12} \\ A_{21} & A_{22}\end{array}\right] \quad B=\left[\begin{array}{c}B_{1} \\ 0\end{array}\right] \quad F C=\left[\begin{array}{ll}F_{1} & 0\end{array}\right]$

where $B_{1}, F_{1} \in \mathbb{R}^{m \times m}$ and both matrices are nonsingular. Furthermore the matrix $A_{22} \in \mathbb{R}^{(n-m) \times(n-m)}$ is stable since the eigenvalues of $A_{22}$ represent the invariant zeros of the triple $(A, B, F C)$ (which are stable by assumption). Further details are given in [15]. In order that $B F C=\Gamma$ holds, it follows that by choice of $F$, the relationship $F_{1} B_{1}=I_{m}$ or equivalently $F_{1}=B_{1}^{-1}$ must hold.

\subsection{Stability criterion for node level system}

Proposition 1 (Node level stability criterion) Consider the node level linear system given in (3) and (4) and suppose the node states are in the canonical representation in (5), then there exists a scalar $\gamma_{0}>0$ such that the local output feedback control law $u=-\gamma F y$, has the property that $(A-$ $\gamma B F C)$ is stable for all $\gamma>\gamma_{0}$.

Proof: Consider the closed loop node level system $(A-$ $\gamma B F C)$, which is $(A-\gamma \Gamma)$ by Assumption 4. Consider the problem of finding a s.p.d matrix $P$ such that

$\mathscr{V}=P(A-\gamma \Gamma)+(A-\gamma \Gamma)^{\mathrm{T}} P<0$

for all $\gamma \geq \gamma_{0}$ for some $\gamma_{0} \geq 0$. This is a sufficient condition for $(A-\bar{\gamma} \Gamma)$ to be stable for all $\gamma>\gamma_{0} \geq 0$. In order for (6) to hold for all $\gamma>\gamma_{0}$ the matrix inequality $\left(P \Gamma+\Gamma^{\mathrm{T}} P\right) \geq 0$ must hold. Suppose for a contradiction that $\left(P \Gamma+\Gamma^{\mathrm{T}} P\right)$ is not semi-positive definite. If this is the case, then the symmetric matrix $\left(P \Gamma+\Gamma^{\mathrm{T}} P\right)$ has a negative eigenvalue $\underline{\lambda}<0$ and an associated eigenvector $v \neq 0$ s.t. $v^{\mathrm{T}}\left(P \Gamma+\Gamma^{\mathrm{T}} \bar{P}\right) v=\underline{\lambda}\|v\|^{2}$. Multiplying $\mathscr{V}$ from (6) on the left and right by $v^{\mathrm{T}}$ and $v$ respectively, it follows

$$
\begin{aligned}
v^{\mathrm{T}} \mathscr{V} v & =v^{\mathrm{T}}\left(P A+A^{\mathrm{T}} P\right) v-\gamma v^{\mathrm{T}}\left(P \Gamma+\Gamma^{\mathrm{T}} P\right) v \\
& =v^{\mathrm{T}}\left(P A+A^{\mathrm{T}} P\right) v-\gamma \underline{\lambda}\|v\|^{2}
\end{aligned}
$$

Since $-\underline{\lambda}\|v\|^{2}>0$, for a sufficiently large value of $\gamma$,

$$
v^{\mathrm{T}} \mathscr{V} v=v^{\mathrm{T}}\left(P A+A^{\mathrm{T}} P\right) v-\gamma \underline{\underline{\lambda}}\|v\|^{2}>0
$$

which contradicts $P(A-\gamma \Gamma)+(A-\gamma \Gamma)^{\mathrm{T}} P<0$ for all $\gamma>\gamma_{0}$. Consequently a necessary condition for (6) to hold is that $\left(P \Gamma+\Gamma^{\mathrm{T}} P\right) \geq 0$. Partition the s.p.d matrix from (6) as

$$
P=\left[\begin{array}{ll}
P_{1} & P_{2} \\
P_{2}^{\mathrm{T}} & P_{3}
\end{array}\right]
$$

where $P_{1} \in \mathbb{R}^{m \times m}$. Making use of Assumption 2:

$$
\left(P \Gamma+\Gamma^{\mathrm{T}} P\right)=\left[\begin{array}{cc}
2 P_{1} & P_{2} \\
P_{2}^{\mathrm{T}} & 0
\end{array}\right]
$$

Therefore a necessary and sufficient condition for $(P \Gamma+$ $\left.\Gamma^{\mathrm{T}} P\right) \geq 0$ is that $P_{2}=0$ and so $P=\mathscr{D} \operatorname{iag}\left\{P_{1}, P_{3}\right\}$. Furthermore choose $P_{1}=\left(B_{1}^{-1}\right)^{\mathrm{T}} B_{1}^{-1}$ (which is quite legitimate since $\left(B_{1}^{-1}\right)^{\mathrm{T}} B_{1}^{-1}>0$ because $\left.\operatorname{det}\left(B_{1}\right) \neq 0\right)$.

It will now be shown that there exists a $\gamma_{0}$ such that (6) holds for all $\gamma>\gamma_{0}$. From the canonical form in (5), by direct computation, $\mathscr{V}$ from (6) has the form

$$
\mathscr{V}=\left[\begin{array}{cc}
P_{1} A_{11}+A_{11}^{\mathrm{T}} P_{1}-2 \gamma P_{1} & P_{1} A_{12}+A_{21}^{\mathrm{T}} P_{3} \\
P_{3} A_{21}+A_{12}^{\mathrm{T}} P_{1} & P_{3} A_{22}+A_{22}^{\mathrm{T}} P_{3}
\end{array}\right]
$$

where $P_{1}=\left(B_{1}^{-1}\right)^{\mathrm{T}} B_{1}^{-1}>0$. Recall that from the minimum phase assumption (Assumption 5), the matrix $A_{22}$ is stable. Hence there exists a $P_{3} \in \mathbf{S}_{+}^{n-m}$, such that

$$
Q_{3}:=P_{3} A_{22}+A_{22}^{\mathrm{T}} P_{3}<0
$$

By the Schur complement [20], $\mathscr{V}<0$ if and only if

$$
2 \gamma P_{1}>P_{1} A_{11}+A_{11}^{\mathrm{T}} P_{1}-\left(P_{1} A_{12}+A_{21}^{\mathrm{T}} P_{3}\right) Q_{3}^{-1}\left(P_{1} A_{12}+A_{21}^{\mathrm{T}} P_{3}\right)^{\mathrm{T}}
$$

Since $P_{1}>0$ this can always be satisfied for a large enough $\gamma$. Let $\gamma_{0}$ be the minimum value of $\gamma$ with respect to the choice of $P_{3}$ and $P_{1}=\left(B_{1}^{-1}\right)^{\mathrm{T}} B_{1}^{-1}$. Then:

$$
P\left(A-\gamma_{0} \Gamma\right)+\left(A-\gamma_{0} \Gamma\right)^{\mathrm{T}} P<0
$$


Furthermore since

$$
\begin{aligned}
\mathscr{V} & =P(A-\gamma \Gamma)+(A-\gamma \Gamma)^{\mathrm{T}} P \\
& =\underbrace{P\left(A-\gamma_{0} \Gamma\right)+\left(A-\gamma_{0} \Gamma\right)^{\mathrm{T}} P}_{<0}+\left(\gamma_{0}-\gamma\right) \underbrace{\left(P \Gamma+\Gamma^{\mathrm{T}} P\right)}_{\geq 0}
\end{aligned}
$$

It follows $\mathscr{V}<0$ for all $\gamma \geq \gamma_{0}$ as required. This completes the proof

Remark 2 If $P=\mathscr{D}$ iag $\left\{P_{1}, P_{3}\right\}$ where $P_{1}=\left(B_{1}^{-1}\right)^{T} B_{1}^{-1}$ and $P_{3} \in \mathbf{S}_{+}^{n-m}$, then by direct computation it can be easily shown that $P B=(F C)^{T}$ The significance of this will be discussed later in the paper.

Remark 3 The minimum value of $\gamma_{0}$ can be found via the LMI optimization problem given below: Minimize $\gamma$ with respect to the s.p.d. matrix $P_{3}$, subject to

$$
\begin{aligned}
{\left[\left(B_{1}^{-1}\right)^{T} B_{1}^{-1} A_{11}+A_{11}^{T}\left(B_{1}^{-1}\right)^{T} B_{1}^{-1}-2 \gamma\left(B_{1}^{-1}\right)^{T} B_{1}^{-1}\right.} & \\
P_{3} A_{21}+A_{12}^{T}\left(B_{1}^{-1}\right)^{T} B_{1}^{-1} & \\
\left.\left(B_{1}^{-1}\right)^{T} B_{1}^{-1} A_{12}+A_{21}^{T} P_{3}\right] & <0 \\
\left.P_{3} A_{22}+A_{22}^{T} P_{3}\right] & \\
P_{3} & <0 \\
\gamma & <0
\end{aligned}
$$

The problem defined in (9) - (11) is a convex optimization problem and can be solved using standard LMI solvers [21].

Remark 4 Proposition 1 can be interpreted as each triple $\left(\left(A-\gamma_{i} B F C\right), B, F C\right)$ is strictly positive real [18] for all $\gamma_{i}>\gamma_{0}$ since there exists a s.p.d matrix $P$ such that

$P\left(A-\gamma_{i} B F C\right)+\left(A-\gamma_{i} B F C\right)^{T} P<0$

and $P B=(F C)^{T}$. This is similar to the constrained Lyapunov problem in [15-17].

\subsection{Static output feedback control}

Proposition 2 (Network level global stabilisation criterion) Consider the network $\mathscr{G}$ consisting of $N$ identical linear systems as given in (3) and (4). Suppose the node states are already in the canonical representation as in (5) and a $\gamma_{0}$ has been computed such that Proposition 1 holds. Then the network $\mathscr{G}$ can be stabilised using a decentralised static output feedback control law $u_{i}=-\gamma_{i} F y_{i}$, if

$$
c L+D_{r}>\gamma_{0} I_{N}
$$

holds where $D_{r}:=\mathscr{D} \operatorname{iag}\left\{\gamma_{1}, \gamma_{2}, \ldots, \gamma_{N}\right\}$ and the $\gamma_{i}$ are the local feedback gains, $c$ is the scalar coupling strength and $V$ the Laplacian matrix of $\mathscr{G}$.

Proof: Substituting for the control law in (3) yields

$$
\dot{x}_{i}=A x_{i}-\gamma_{i} \Gamma x_{i}-\sum_{j=1}^{N} c L_{i j} \Gamma x_{j}
$$

$y_{i}=C x_{i}$ since by Assumption 4, $B F C=\Gamma$. By simple algebraic manipulation, equation (13) can be conveniently written as

$\dot{x}_{i}=A x_{i}-\sum_{j=1}^{N} c \tilde{L}_{i j} \Gamma x_{j}$

where $\tilde{L}:=L+D_{r} / c$. The dynamics of the overall network in (15) can conveniently be written as

$\dot{x}=\left(I_{N} \otimes A-c(\tilde{L} \otimes \Gamma)\right) x$

Note that by construction, $\tilde{L}$ is dependent on the control gains $\gamma_{i}, i=1 \ldots N$. Also by construction $\tilde{L}$ is a symmetric matrix since both $L$ and $D_{r}$ are symmetric. By spectral decomposition the symmetric matrix $\tilde{L}$ can be written as

$\tilde{L}=V D V^{\mathrm{T}}$

where the orthogonal matrix $V \in \mathbb{R}^{N \times N}$ is formed from the eigenvectors of $\tilde{L}$, and $D:=\mathscr{D} \operatorname{iag}\left(d_{1}, d_{2}, . ., d_{i}, \ldots, d_{N}\right)$. Define a co-ordinate transformation $T: x \mapsto z:=T x$, where

$T=\left(V^{\mathrm{T}} \otimes I_{n}\right)$

and $V$ is the orthogonal matrix from the spectral decomposition in (17). The transformation matrix $T$ is an orthogonal transformation since since $V$ is orthogonal. Applying the transformation given in (18) to (16), after algebraic manipulations making use of the Kronecker identities:

$\dot{z}=\left(\left(I_{N} \otimes A\right)-c(D \otimes \Gamma)\right) z$

This structure enables a static output feedback control problem for stabilisation of the network to be investigated by considering the individual node level dynamics since (19) can be written as $\dot{z}_{i}=\left(A-c d_{i} \Gamma\right) z_{i}$ for $i=1 \ldots N$ where $z=\mathscr{C o l}\left(z_{1}, z_{2}, \ldots z_{N}\right)$ because of the diagonal nature of $D$. It follows from Proposition 1 that if

$$
c d_{i}>\gamma_{0}, \quad \forall i=1 \ldots N
$$

then $\left(A-c d_{i} \Gamma\right)$ is stable and furthermore there exists a s.p.d. $P$ such that, for nodes $i=1 \ldots N$, the following strict matrix inequality holds: $P\left(A-c d_{i} \Gamma\right)+\left(A-c d_{i} \Gamma\right)^{\mathrm{T}} P<0$. Since the $d_{i}$ are the eigenvalues of $\left(L+D_{r} / c\right)$ if

$\left(c L+D_{r}\right)>\gamma_{0} I_{N}$

then $d_{i}>\frac{\gamma_{0}}{c}$ for $i=1 \ldots N$. Thus the network stability problem is to choose the gains $\gamma_{i}$ for all $i=1 \ldots N$ such that (20) holds. This completes the proof.

Remark 5 In [9] a separation of the Laplacian is achieved and a condition is introduced incorporating the concept of a passivity degree. However, the methodology in this paper has origins in a more classical control approach. Moreover, a systematic approach using LMIs has been developed.

Remark 6 The orthogonal transformation in (18) provides a decoupled structure and restricts the analysis to the node level dynamics of the network. However because information about L is used to compute $D_{r}$, there is an implicit assumption that the network is fixed. Thus the approach proposed in this paper is not applicable to dynamic ad-hoc networks where nodes are added/deleted. 
When $D_{r}$ is full rank, it implies that control signals are injected in every node of the network. The objective of the paper is to obtain (global) stabilisation with most of the $\gamma_{i}$ entries as zero. The solution to the problem is not trivial and imposes a rank constraint on this matrix.

\subsection{LMI formulation}

The problem of designing the decentralised output feedback control laws is tackled as a two stage LMI optimization problem: the first one as the convex LMI optimisation problem as defined in (9) - (11). This is a well defined generalized eigenvalue problem and can be solved using any LMI solver. Once $\gamma_{0}$ has been computed, a second optimization problem can be solved involving the matrix $D_{r}$ which represents the output feedback gains injected at each node.

A non-convex approach can be adopted to try to minimize the number of nodes at which control is applied. The numerical algorithm is required to find a solution to a rank constrained LMI problem: see [22] and the references therein. This optimization problem can be cast as:

Minimize Trace $\left(D_{r}\right)$ with respect to $\gamma_{1}, \gamma_{2} \ldots \gamma_{N}$, subject to:

$$
\begin{aligned}
c L+D_{r} & >\gamma_{0} I_{N} \\
D_{r} & \geq 0 \\
\operatorname{rank}\left(D_{r}\right) & \leq r
\end{aligned}
$$

where the positive integer $r$ is chosen by the designer.

Such a problem is generally hard to solve; however there exist algorithms, such as LMIRank [23]. LMIRank can be called using YALMIP [24], a MATLAB toolbox for rapid prototyping of optimization algorithms. However, LMIRank does not support objective functions, and only solves feasibility problems. However the objective function can be minimized using an outer loop bisection algorithm. The rank computations being inherently hard from a numerical view point, means occasionally the actual rank obtained is higher than $r$, even though LMIRank claims feasibility. Such situations require tuning of the tolerances of the solver. These are known, reported issues associated with the solver [22,23].

If the $l$ out of $N$ nodes at which the control is applied are decided upon a-priori (based on the designer's intuition) then the problem can be posed as:

Minimize Trace $\left(D_{r}\right)$ subject to (21)-(22).

This represents a convex optimization problem and so can be tackled using LMI solvers [21]. In the LMI optimisation problem, $N-l$ entries of $D_{r}$ matrices are fixed as zeros to ensure no control input injection in the respective channels. This is a sufficiency condition. In case of full node output feedback injection, the static output feedback gain matrix $D_{r}$ is full rank, which implies that control signals are injected in every node of the network. In the case of partial node output feedback injection to $l$ nodes out of $N$, the static output feedback gain matrix $D_{r}$ is of rank $l$.

\section{Remark 7}

- The trace minimization attempts to minimize the use of control effort by choosing 'small' feedback gains.
- This approach is reliant on the designer choosing a-priori the nodes in which to inject control signals - which will be difficult for large networks.

\section{Network stabilisation: Nonlinear extension}

The results discussed so far pertain to the linear system in (3) and (4). This is now extended to the nonlinear case in (1) and (2). The closed-loop dynamics of the network including nonlinearities/uncertainties can be represented as:

$\dot{x}=\left(I_{N} \otimes A\right) x-c(\tilde{L} \otimes \Gamma) x+f(x)$

where $f(x)=\mathscr{C} o l\left(f_{1}\left(x_{1}\right), \ldots f_{N}\left(x_{N}\right)\right)$ represents the vector of nonlinearities.

Assumption 6 The nonlinearities satisfy $f_{i}\left(x_{i}\right)=B \xi_{i}\left(y_{i}\right)$ for $i=1 \ldots N$ for some functions of the states $\xi_{i}\left(y_{i}\right)$, where

$\left(F y_{i}\right)^{T}\left(\xi_{i}\right) \leq 0$

is satisfied for all $x_{i}$ (where $y_{i}$ is thought of as $C x_{i}$ ).

Equation (25) represents a sector condition on the nonlinearity $\xi_{i}\left(x_{i}\right)$. Define $\xi=\mathscr{C}$ ol $\left(\xi_{1}, \ldots, \xi_{N}\right)$. As argued earlier, the triples $\left(\left(A-\gamma_{i} B F C\right), B, F C\right)$ are strictly positive real for $\gamma_{i}>\gamma_{0}$ i.e. there exists a $P$ such that

$P\left(A-\gamma_{i} B F C\right)+\left(A-\gamma_{i} B F C\right)^{\mathrm{T}} P<0$

and $P B=(F C)^{\mathrm{T}}$. Define matrices $\mathbb{B}:=I_{N} \otimes B, \mathbb{C}:=I_{N} \otimes C$, $\mathbb{F}:=I_{N} \otimes F, \mathbb{P}:=I_{N} \otimes P$ and $y=\mathscr{C}$ ol $\left(y_{1}, \ldots y_{N}\right)$. Then

$$
(\mathbb{F} y)^{\mathrm{T}} \xi=\sum_{i=1}^{N}(F y)^{\mathrm{T}} \xi_{i} \leq 0
$$

since from Assumption 6, the nonlinearities satisfy $\left(F y_{i}\right)^{\mathrm{T}} \xi_{i} \leq 0$ for $i=1 \ldots N$. Also notice that

$$
\mathbb{P B}=(\mathbb{F} \mathbb{C})^{\mathrm{T}}
$$

since $P B=(F C)^{\mathrm{T}}$ and

$$
\mathbb{P}\left(I_{N} \otimes A-c(\tilde{L} \otimes \Gamma)\right)+\left(I_{N} \otimes A-c(\tilde{L} \otimes \Gamma)\right)^{\mathrm{T}} \mathbb{P}<0
$$

It follows that $\mathbb{V}(x)=x^{\mathrm{T}} \mathbb{P} x$ is a Lyapunov function for the nonlinear system in (24) written as

$\dot{x}=\left(I_{N} \otimes A\right) x-c(\tilde{L} \otimes \Gamma) x+\left(I_{N} \otimes B\right) \xi$

\section{Network synchronisation as a stabilisation problem}

Consider the synchronisation problem, where the objective is to drive the states of the dynamical network given in (1) and (2) to a desired orbit. The desired orbit is $x=s\left(t, t_{0}, x_{0}\right) \in \mathbb{R}^{n}$ with $x_{0} \in \mathbb{R}^{n}$. Write the orbit more concisely as $s(t)$ and suppose it is generated by a virtual master system, a leader system, or even the solution of a stand alone node of the complex network. Note that $s(t)$ can be an equilibrium point, a periodic orbit, or even a chaotic orbit in the phase space. 
Consider the scenario of synchronising the network to a desired orbit generated by a virtual master node. Let the dynamics of the virtual master node be:

$$
\begin{aligned}
\dot{s} & =A s+B \xi\left(y_{s}\right) \\
y_{s} & =C s
\end{aligned}
$$

where $B \xi\left(y_{s}\right)=f(s)$. The solution to (28)-(29) provides the $s(t)$ to which the states of the network are to be synchronised to. To develop a controller to induce synchronicity, as in $[3,7,25]$, define an error vector $e_{i}(t):=x_{i}(t)-s(t)$ for $i=$ $1 \ldots N$. Subtracting the virtual master node dynamics in (28) from equation (1) yields

$$
\begin{aligned}
\dot{e}_{i} & =A e_{i}+B u_{i}-\sum_{j=1}^{N} c L_{i j} \Gamma e_{j}+B\left(\xi_{i}\left(y_{i}\right)-\xi\left(y_{s}\right)\right) \\
e_{y_{i}} & =C e_{i}
\end{aligned}
$$

To obtain this structure, the row sum property of the Laplacian matrix $L$ has been exploited i.e $\sum_{j=1}^{N} c L_{i j} \Gamma s=$ $c\left(\sum_{j=1}^{N} L_{i j}\right) \Gamma s=0$ since $\sum_{j=1}^{N} L_{i j}=0$. Write the term $\xi_{i}\left(y_{i}\right)-\xi\left(y_{s}\right)$ in (30) in the form

$\psi_{i}\left(e_{y_{i}}, y_{s}\right):=\xi_{i}\left(e_{y_{i}}+y_{s}\right)-\xi\left(y_{s}\right)$

\section{Assumption 7 The nonlinearities $\psi_{i}\left(e_{y_{i}}, y_{s}\right)$ satisfy}

$$
\left(F e_{y_{i}}\right)^{T} \psi_{i}\left(e_{y_{i}}, y_{s}\right) \leq 0
$$

for all $e_{y_{i}}$ and $y_{s}$.

Substituting (32) in the error dynamics (30)

$$
\begin{aligned}
\dot{e}_{i} & =A e_{i}+B u_{i}-\sum_{j=1}^{N} c L_{i j} \Gamma e_{j}+B \psi_{i}\left(e_{y_{i}}, y_{s}\right) \\
e_{y_{i}} & =C e_{i}
\end{aligned}
$$

Using Assumption 7 and the results in Section 4, the network error system (35) is asymptotically stable. Since $e_{i} \rightarrow 0$, it follows $x_{i} \rightarrow s$ and synchronization is achieved.

\section{Numerical example}

To demonstrate the theory developed in this paper, a network of Chua oscillators [25] will be utilized. Consider an arbitrary graph $\mathscr{G}$ representing a network of dynamical systems consisting of 75 nodes. The individual nodes are assumed to be identical. The individual node dynamics are:

$\dot{x}_{i}=A x_{i}+B \xi_{i}\left(y_{i}\right)-c \sum_{j=1}^{N} L_{i j} \Gamma x_{j}+B u_{i}$
$y_{i}=C x_{i}$

where

$A=\left[\begin{array}{ccc}-a m_{1} & a & 0 \\ 1 & -1 & 1 \\ 0 & -b & 0\end{array}\right], B=\left[\begin{array}{c}-a\left(m_{0}-m_{1}\right) \\ 0 \\ 0\end{array}\right], C^{\mathrm{T}}=\left[\begin{array}{l}1 \\ 0 \\ 0\end{array}\right]$

Note that a Lur'e type representation for the network of Chua's oscillators has been adopted. The nonlinearity is $\xi_{i}\left(y_{i}\right)=\frac{1}{2}\left(\left|y_{i}+\rho\right|-\left|y_{i}-\rho\right|\right)$, which has a sector bound $[0,1]$ and is monotonic. The chosen values of the parameters are $a=9, b=14.286, \rho=1, m_{0}=-1 / 7, m_{1}=2 / 7$ in order to attain the double scroll attractor [25]. In the network, the coupling strength $c$ is assumed to be identical and fixed as 0.5 . The matrix $\Gamma=\mathscr{D} \operatorname{iag}\{1,0,0\}$, which is consistent with Assumption 2. Consider now a virtual master system described in (28). Following the arguments in the previous section, and adding and subtracting a $B e_{y_{i}}$ term to the differential equations representing each node, the error dynamics for synchronisation are given by:

$$
\begin{aligned}
\dot{e}_{i} & =\bar{A} e_{i}+B \bar{\psi}_{i}\left(e_{y_{i}}, y_{s}\right)-c \sum_{j=1}^{N} L_{i j} \Gamma e_{j}+B u_{i} \\
e_{y_{i}} & =C e_{i}
\end{aligned}
$$

where $\bar{A}:=A+B C$ and

$$
\begin{aligned}
\bar{\psi}_{i}\left(e_{y_{i}}, y_{s}\right): & =\left(\xi_{i}\left(y_{s}+e_{y_{i}}\right)-\xi_{i}\left(y_{s}\right)\right)-e_{y_{i}} \\
& =\operatorname{sat}\left(y_{s}+e_{y_{i}}\right)-\operatorname{sat}\left(y_{s}\right)-e_{y_{i}}
\end{aligned}
$$

where sat $(\cdot)$ represents the saturation function. It follows

$$
\bar{\psi}_{i}\left(e_{y_{i}}, y_{s}\right) F e_{y_{i}} \leq 0
$$

for all $e_{y_{i}}, y_{s} \in \mathbb{R}$ and $F \in \mathbb{R}^{+}$from the monotonicity properties of the saturation function. This is the sector constraint in Assumption 6 and so the results from the previous section can now be invoked based on the triple $(\bar{A}, B, C)$.

Four different case studies are reported in this paper: solutions with rank 5, 7, 9 and 75 (full node control). In the LMI's, a stability margin has been selected as 0.005 for all cases. It is easy to check $(\bar{A}, B, C)$ satisfies Assumption 3.

Also choosing $F:=-1 / a\left(m_{0}-m_{1}\right)=0.2593$ ensures Assumption 4 holds. It can also be verified that $(\bar{A}, B, C)$ is minimum phase and $C B \neq 0$, and the pair $(\bar{A}, B)$ is controllable. Hence, Assumption 5 is also satisfied. By solving the necessary LMI conditions, in (11)-(12), $\gamma_{0}$ is obtained as 10.6463. In the second stage of the optimization, the rank constraints are imposed for the different cases and the local decentralised output feedback gains are obtained such that Proposition 14 holds.

Consider Case I with rank 5 (the minimum rank obtained for which the network is stabilisable). The LMIRank solver gives a solution which corresponds to nodes 2,6,21,38 and 67, having nonzero feedback. The output feedback control gains for these nodes are [549.22, 536.21, 535.24, 541.33, 549.57]. The nodes in which control signals have been injected and the respective control gains are provided in Table 6 for the first three cases. It is interesting to note that the nodes utilised in case I have also been utilised in Case II and Case III. In Table 6 the average value of the control gains in the case of rank 5 is larger than that of rank 7 and rank 9. When more nodes are used to inject the output feedback control signals, the smaller the gains. In the case of full node control, the gain at each individual node is only 10.6463 .

The simulation results for case I are shown in Fig. 1. The subplots in Fig. 1 represent the time response of states $x_{1}, x_{2}$ 


\begin{tabular}{|l|c|c|c|c|c|c|c|c|c|c|}
\hline Case & Parameter & \multicolumn{7}{|c|}{ Values } \\
\hline I & $\begin{array}{l}\text { Node } \\
\text { Gain }\end{array}$ & $\begin{array}{c}2 \\
549.22\end{array}$ & $\begin{array}{c}6 \\
536.21\end{array}$ & $\begin{array}{c}21 \\
535.24\end{array}$ & $\begin{array}{c}38 \\
541.33\end{array}$ & $\begin{array}{c}67 \\
549.57\end{array}$ & & & & \\
\hline \multirow{2}{*}{ II } & Node & 2 & 6 & 8 & 21 & 38 & 53 & 67 & & \\
& Gain & 227.08 & 223.60 & 221.93 & 221.54 & 226.49 & 223.66 & 227.22 & & \\
\hline \multirow{2}{*}{ III } & Node & 2 & 3 & 6 & 8 & 21 & 38 & 53 & 67 & 74 \\
& Gain & 143.71 & 142.44 & 143.88 & 142.23 & 141.42 & 143.55 & 142.29 & 143.76 & 141.32 \\
\hline
\end{tabular}

Table 6: Feedback gains for different pinning strategies

and $x_{3}$ respectively. The black dashed line (line width kept double) represents the time responses of the master node, whereas the green line shows the time responses of a node, picked randomly from the 70 nodes in which no control signal is applied. Fig. 2 shows the synchronisation to double scroll attractor dynamics. Note that the initial condition of the phase plots corresponds to a time $\mathrm{t}=10$ seconds, for clarity. The upper subplots in Fig. 2 demonstrate the double scroll dynamics of the master node, whereas the bottom subfigures show that of the randomly picked node. To visualize the synchronisation, the mean square error in the network, $\sum_{i=1}^{N} e_{i, j}(t)^{2}$ for $j=1 \ldots 3$ where $e_{i, j(t)}$ is the $j$ th component of $e_{i}(t)$, has been used. Fig. 3 shows the mean squared error becomes zero within 15 secs. The results are almost identical in all the other cases; however the control effort required by the minimum rank stabilisation case is higher compared to the full node case.

\section{Conclusions}

In this paper, the stabilisation and synchronisation of the network dynamical system is achieved using a decentralised output feedback strategy. The four block representation and associated invariance structure of the networked dynamical systems have been exploited in the problem formulation. The stabilisation problem with the minimum number of nodes 'pinned' is formulated as a rank constrained linear matrix inequality problem. The non-convex rank constraint emanating from the attempt to use the minimum number of nodes for control signal injection is solved using LMIRank methods. This paper has also demonstrated how to exploit the notion of positive realness in the closed-loop node level dynamics so that the formulation can easily handle a wide class of nonlinearities/uncertainties satisfying sector conditions. The synchronisation problem is also posed as a stabilisation problem. The theory is applied to a relatively complex academic example of a network of Chua oscillators and its efficacy is demonstrated.

\section{References}

[1] V. Kumar, N. Leonard, and A.S. Morse, (Eds.), Cooperative control, Springer, LNCIS, vol. 309, 2005.

[2] W. Ren and R. W. Beard, Distributed consensus in multi-vehicle cooperative control, Springer, Lecture notes in communication and control engineering series, 2007.

[3] C. W. Wu, Synchronisation of complex networks of nonlinear dynamical systems, World scientific publishing company, 2007.
[4] M. Newman, The structure and dynamics of networks, Princeton, NJ: Princeton university press, 2006.

[5] D.J. Watts and S.H. Strogatz, "Collective dynamics of small-world networks," Nature, vol. 393, pp. 440-442, 1998

[6] D.J. Watts, "Small-worlds: the dynamics of networks between order and randomness," Princeton, NJ: Princeton University Press, 1999.

[7] X.F. Wang and G. Chen, "Complex networks: small-world, scale-free and beyond," IEEE Circ. Syst Mag, vol. 3, pp. 6-20, 2003.

[8] X.F. Wang and G. Chen, "Pinning control of scale-free network," Physica A, vol. 310, pp. 521-531, 2002.

[9] X. Ji and G. Chen, "On the V-stability of complex dynamical networks," Automatica, vol. 43, pp. 1049-1057, 2007.

[10] M. Arcak, "Passivity as a design tool for group coordination," IEEE Trans. Autom. Control, vol. 52, pp. 1380-1390, 2007.

[11] J. A. Fax and R. M. Murray, "Information flow and cooperative control of vehicle formations," IEEE Trans. Autom. Control, vol. 49, pp. 1465-1476, 2004.

[12] L. Moreau, "Stability of multiagent systems with time dependent communication links," IEEE Trans. Autom. Control, vol. 50, pp. 169-182, 2005.

[13] Z. Lin, B. Francis, M. Maggiore, "State agreement for continuous time coupled nonlinear systems," SIAM J. Control and Optim., vol. 46, pp. 288-307, 2007.

[14] X. G. Yan, C. Edwards and S. K. Spurgeon, "Decentralized robust sliding model control for a class of nonlinear interconnected systems by static output feedback," Automatica, vol. 40, pp. 613-620, 2004.

[15] C. Edwards, X. G. Yan and S. K. Spurgeon, "On the solvability of the constrained Lyapunov problem," IEEE Trans. Autom. Control, vol. 52, pp. 1975-1981, 2007.

[16] A.R. Galimidi and B.R. Barmish, "The constrained Lyapunov problem and its application to robust output feedback stabilisation," IEEE Trans. Autom. Control, vol. 31, pp. 410-418, 1986.

[17] G. Gu."Stabilizability conditions of multivariable uncertain systems via output feedback control," IEEE Trans. Autom. Control, vol. 35, pp. 925-927, 1990.

[18] H. Khalil, Nonlinear systems, Prentice Hall, $3^{\text {rd }}$ edition, NY., 2002.

[19] G. Royle and C. Godsil, Algebraic graph theory, Springer Verlag, New York, 2001.

[20] S. Boyd, L. El Ghaoui, E. Feron, and V. Balakrishnan, Linear Matrix Inequalities in system and control theory, SIAM, 1994.

[21] P. Gahinet, A. Nemirovski, A.J. Laub, and M.I. Chilal, LMI control tool box, The MathWorks Inc., 1995.

[22] R. Orsi, U. Helmke, and J.B. Moore, "A Newton-like method for solving rank constrained linear matrix inequalities," Automatica, vol. 42, pp. 1875-1882, 2006.

[23] R. Orsi, "LMIRank: software for rank constrained LMI problems," Available from $\{$ http://rsise.anu.edu.au/robert/lmirank/\}, 2005.

[24] J. Laofberg, "YALMIP: A toolbox for modeling and optimization in MATLAB," In Proc. CACSD Conf., Taiwan, 2004, $\{$ http://control.ee.ethz.ch/ joloef/yalmip.php\}. 
[25] J. A. K. Suykens, P. F. Curran, and L. O. Chua, "Robust synthesis for master - slave synchronisation of Lur'e systems," IEEE Trans Circuits Syst-I, vol. 46, pp. 841-850, 1999.

[26] J.T. Spooner and K.M. Passino, "Adaptive control of a class of decentralized nonlinear systems", IEEE Trans. on Automatic Cont., vol. 41, pp. 280-284, 1996.

[27] V. Gazi and K. M. Passino, "Decentralized output regulation of a class of nonlinear systems," Int. J. Cont., vol. 79, pp. 1512-1522, 2006 .

[28] X.-G. Yan and L. Xie, "Reduced-order control for a class of nonlinear similar interconnected systems with mismatched uncertainty," Automatica, vol. 39, pp 91-99, 2003.

[29] X.-G. Yan, J.-J. Wang, X.-Y. Lu and S.-Y. Zhang, "Decentralized output feedback robust stabilization for a class of nonlinear interconnected systems with similarity," IEEE Trans. Auto. Contr., vol. 43, pp. 294-299, 2005.

[30] V. Gazi, "Formation control of a multi-agent system using nonlinear servomechanism", Int. J. Cont., vol. 78, pp. 554-565, 2005.

[31] S-J. Chung, U. Ahsun and J.-J. E. Slotine, "Application of Synchronization to Formation Flying Spacecraft: Lagrangian Approach," AIAA Journal of Guidance Control and Dynamics, vol. 32, pp. 512 - 526, 2009.

[32] J. Zhou, J. Lu and J. Lu, "Adaptive synchronization of an uncertain complex dynamical network," IEEE Trans. Auto. Contr., vol. 51, pp. 652-656, 2006.

[33] L. Bakule, "Decentralized control: An overview," Annual Reviews in Control, vol. 32, pp. 87-98, 2008.
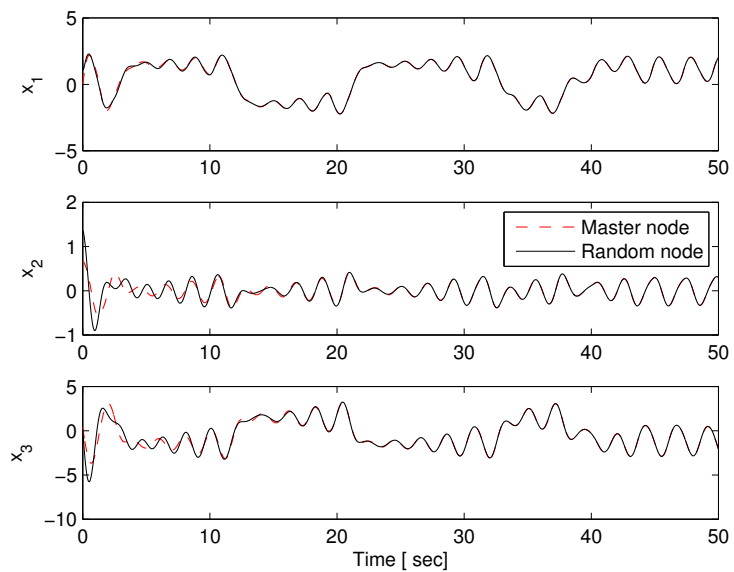

Fig. 1. State synchronisation of a random node
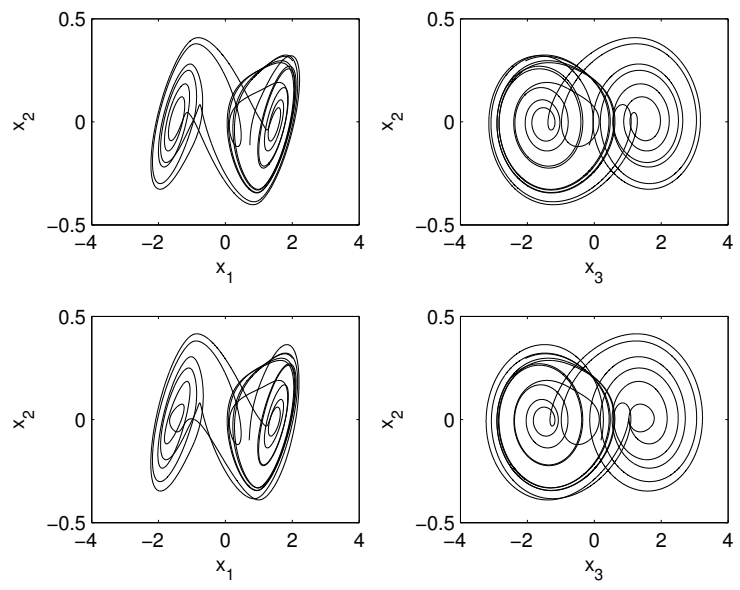

Fig. 2. Double-scroll dynamics
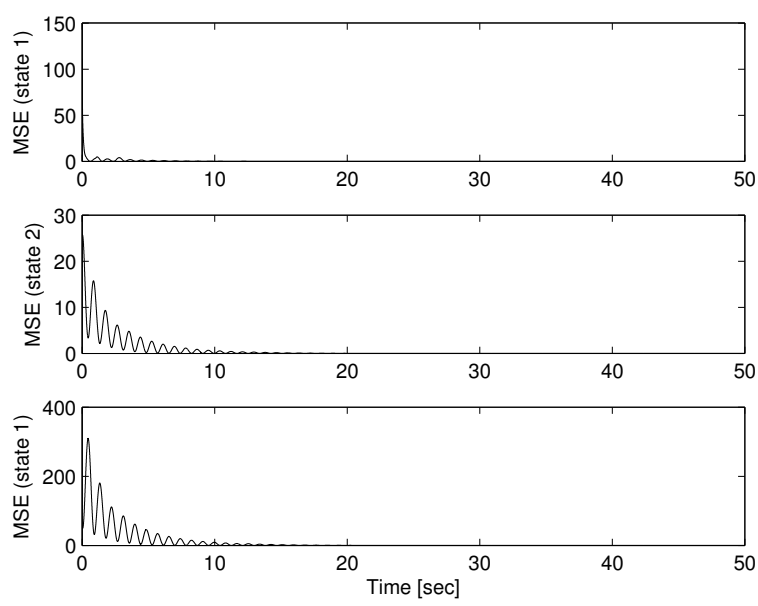

Fig. 3. Mean square error convergence of states of 75 nodes of the network, Case I (rank 5) 